\section{Teaching Case Report}

\section{The role of the Coombs test}

\section{in evaluating hemolysis in}

\section{adults}

The Case: A 72-year-old man with chronic lymphocytic leukemia presents to the emergency department with a 3month history of shortness of breath on exertion, lethargy, pallor and scleral icterus. Laboratory test results reveal a hemoglobin level of $90 \mathrm{~g} / \mathrm{L}$ (normal range $130-170 \mathrm{~g} / \mathrm{L}$ ), and a type and screen is ordered. The blood bank physician notifies the attending doctor that the patient has a panagglutinating IgG antibody with positive autocontrol and suggests ordering a direct antiglobulin test and other biochemical markers of hemolysis. How should the emergency doctor proceed?

The antiglobulin test was first described by Coombs in 1945 and is often referred to as the Coombs test. It is performed to detect either erythrocyte- directed IgG in plasma or IgG or complement coating on the surface of circulating erythrocytes. Neither of these molecules can cause direct agglutination of erythrocytes, so, to detect their presence, monoclonal antihuman globulin (AHG) with specificity for IgG or various complement proteins is added to a suspension of erythrocytes. The AHG reagent is sufficiently large to cause agglutination of erythrocytes that are coated with IgG or complement. Fig. I illustrates the steps of the direct antiglobulin test (DAT), which detects in vivo binding of either IgG or comple-

\title{
Direct Antiglobulin Test
}
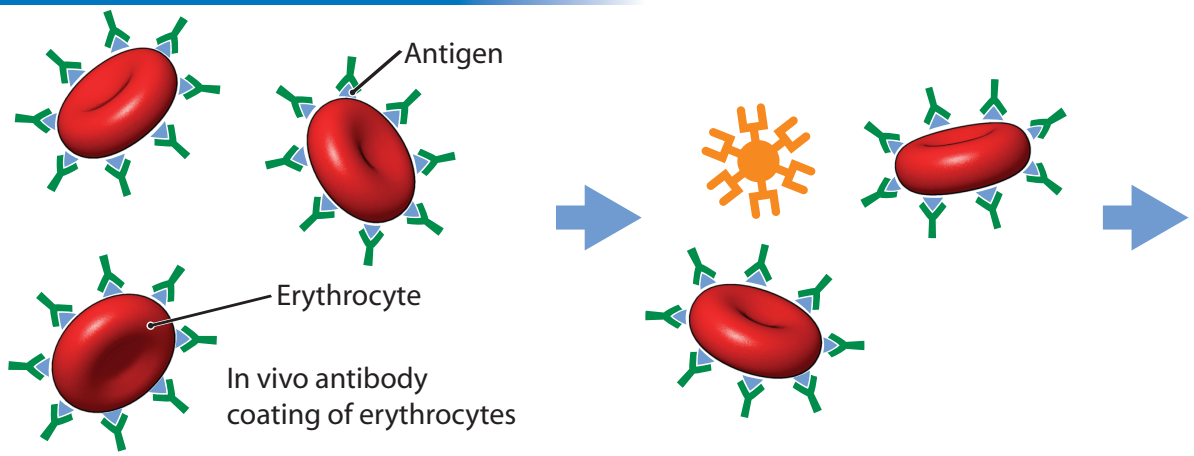

Anti-lgG AHG reagent added after erythrocytes are washed

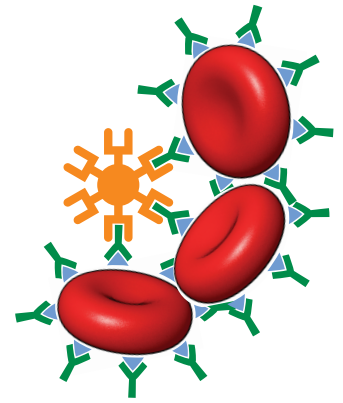

AHG reagent causes IgG-coated erythrocytes to agglutinate

Indirect Antiglobulin Test
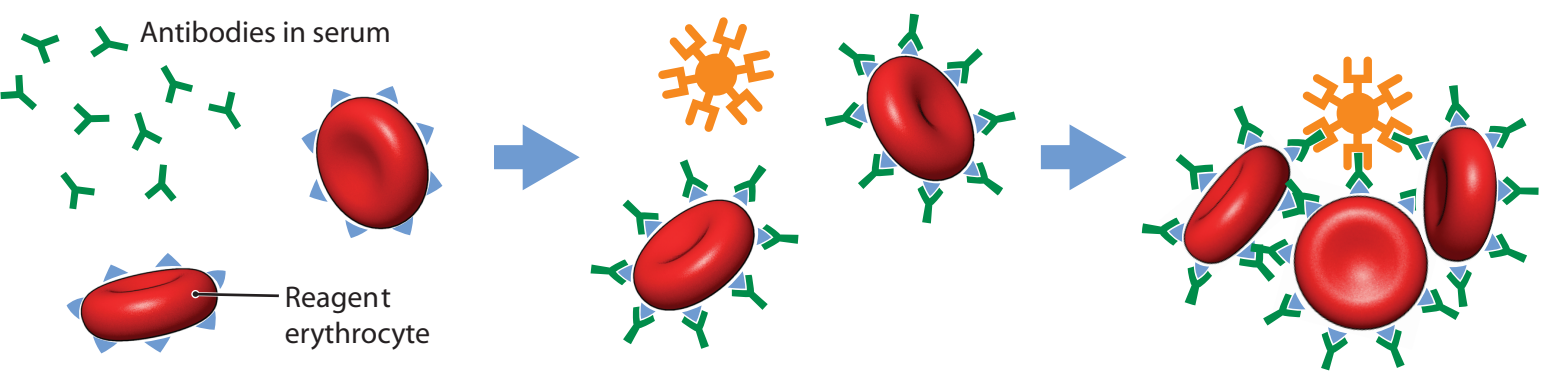

Fig. 1: The direct antiglobulin test (DAT) and indirect antiglobulin test (IAT). AHG = antihuman globulin. A. The DAT reflects in vivo antibody sensitization of erythrocytes. Erythrocytes are washed to remove any unbound antibodies, and anti-IgG AHG reagent is then added. IgG antibodies cannot cause direct erythrocyte agglutination, but if the erythrocytes are coated with IgG antibodies, the AHG reagent will cause them to agglutinate. This test can also be performed using anti-complement AHG reagent. If IgG antibodies are present, they can be eluted off the erythrocytes for specificity determination. B. The IAT is used to detect the presence of IgG antibodies in serum (in vitro sensitization). Reagent erythrocytes are incubated in the presence of serum that potentially contains antibodies. If antibodies are present, they bind to their target antigens on the reagent erythrocytes. After the incubation period the erythrocytes are washed to remove unbound antibodies. Anti-IgG AHG reagent is added and will cause IgG-coated erythrocytes to agglutinate. 
ment to erythrocyte antigens, and the indirect antiglobulin test (IAT), which detects serum antibodies that bind to erythrocytes during an incubation step.

\section{Clinical use of the DAT}

The DAT is useful in differentiating immune-mediated hemolysis from hemolysis caused by other factors (Box I). It is important to note that a small but significant number of patients have a positive DAT result without shortened erythrocyte survival; a positive DAT result without evidence of hemolysis has been reported in I:I-I4 000 healthy blood donors and $\mathrm{I} \%-\mathrm{I} 5 \%$ of in-hospital patients. ${ }^{1,2}$ A positive DAT result in the absence of hemolysis can also occur in certain circumstances (Box I). In these disease conditions, dysregulation of the immune system or a generalized inflammatory state leads to nonspecific adsorption of antibodies to the erythrocyte membrane. These IgG molecules are not immunologically bound to the erythrocyte membrane (i.e., they do not recognize and bind to specific erythrocyte antigens) and do not usually cause hemolysis. An eluate prepared from DAT-positive erythrocytes can determine whether the antibodies were immunologically or nonspecifically attached to these cells.

When a DAT result is positive, it is important to determine whether there is laboratory (Box 2) or clinical evidence of hemolysis. If there is no evidence of hemolysis, no additional investigations are necessary.

However, if there is evidence of hemolysis, the physician needs to explain the cause of the positive test result. One of the most common causes is autoimmune hemolytic anemia (AIHA). AIHA is usually classified on the basis of the thermal amplitude of the autoantibody; warm AIHA is usually caused by an IgG antibody, whereas cold AIHA is typically caused by an IgM antibody that fixes complement to the surface of the erythrocyte. In the former case, the DAT result is positive with anti-IgG AHG reagent, and in the latter, reactiv-

\section{Box 1: Differential diagnosis of hemolysis}

Conditions associated with hemolysis and a positive DAT result

- Hemolytic disease of the newborn

- Drug-induced hemolytic anemias

- Acute hemolytic transfusion reaction

- Delayed hemolytic transfusion reaction

- Autoimmune hemolytic anemia (warm autoimmune hemolytic anemia, cold agglutinin syndrome, paroxysmal cold hemoglobinuria, mixed-type autoimmune hemolytic anemia)

Conditions associated with a positive DAT result, with or without hemolysis

- Exogenous immune globulin administration

- Recent hematopoietic stem-cell transplantation

- Recent solid organ transplantation

- Systemic lupus erythematosus

- Infectious mononucleosis

- Some hematologic diseases, including lymphoproliferative diseases

Conditions associated with hemolysis and a negative DAT result

- Microangiopathic hemolytic anemias (thrombotic thrombocytopenic purpura, disseminated intravascular coagulation)

- Hypersplenism

- Liver disease

- Hemoglobinopathies (sickle cell disease, thalassemia)

- Erythrocyte membranopathies (spherocytosis)

- Deficiencies of erythrocyte enzymes (G-6-PD deficiency, pyruvate kinase deficiency)

- Infectious diseases (Clostridium difficile infection)

- Erythrocyte trauma (mechanical heart valves, improper use of blood warmers) ity with the anti-complement AHG reagent is present (anti-IgM AHG reagents are not readily available). The antibody screen, as part of a type and screen, is a useful way to begin differentiating warm from cold AIHA.

Many pharmacologic agents have been associated with a positive DAT result, with or without hemolytic anemia. Many drugs bind to the membranes of circulating cells; antibodies elicited by these medications may be directed either against a combination of the drug and certain membrane components or against epitopes of the drug molecule that are bound tightly to the erythrocyte surface. Hemolytic anemias associated with these drugs (including penicillins and other antibiotics) frequently resolve once the agent is discontinued and cleared from the circulation. Other medications have been associated with a hemolytic anemia that persists even after the drug is cleared. These drugs, the archetype of which is $\alpha$-methyldopa, are thought to cause hemolytic anemia by inducing the production of anti-erythrocyte autoantibodies that do not require the presence of the drug to bind to erythrocytes.

If the patient has received a transfusion within the previous 3 months, a positive DAT result may indicate alloimmunization to an antigen on the transfused cells that is not present on the recipient's own erythrocytes. In this case only the donor's antigen-positive cells, which stimulated the recipient's immune response, would be coated with the newly formed antibody. The recipient's autologous cells, which lack the antigen, would not be agglutinated by anti-IgG reagents nor hemolyzed by this antibody. An unexpected erythrocyte antibody might appear as early as 7-Io days after transfusion, or earlier if the patient was previously alloimmunized and is re-exposed to the same antigen in a subsequent transfusion.

A less common cause of a positive DAT result is recent administration of intravenous immune globulin, which can contain antibodies directed against $\mathrm{ABO}, \mathrm{Rh}$ or other erythrocyte antigens. The titre of these passively acquired antibodies diminishes over time, and the DAT result will eventually become negative. In patients who have undergone 
Box 2: Biochemical evidence of hemolysis

- Hemoglobinuria

Elevated

- Reticulocyte count

- Lactate dehydrogenase level

- Unconjugated bilirubin level

Decreased

- Hemoglobin level

- Haptoglobin level

either recent hematopoietic stem cell transplantation or solid organ transplantation, donor lymphocytes accompanying the allograft organ or stem cells may produce antibodies with specificity for recipient erythrocyte antigens. In these latter cases, the extent of the chimerism between the antibody-producing lymphocytes and the recipient will dictate how long the DAT result remains positive.

\section{The indirect antiglobulin test and its clinical applications}

The IAT is used by the blood bank to detect unexpected erythrocyte antibodies in the patient's serum or plasma. The IAT is the final phase of the anti- body screen and serologic crossmatch procedures. In an antibody screen the recipient's serum is incubated with 2 or 3 different type $\mathrm{O}$ erythrocytes that express clinically significant antigens. This erythrocyte and serum mixture is then incubated with anti-IgG AHG and observed for agglutination. If agglutination occurs, the screen is considered positive, and further testing is performed to determine the specificity of the IgG antibody. The serologic crossmatch procedure is also an IAT procedure and is performed on patients with unexpected erythrocyte antibodies to verify that the potential donor erythrocyte unit lacks the antigen corresponding to the recipient's antibody. In this procedure, the patient's serum is incubated with erythrocytes from a potential erythrocyte donor unit, and antiIgG reagent is added. Agglutination would indicate an incompatibility between the donor erythrocytes and the recipient's IgG antibody. Thus the results of the IAT reflect in vitro erythrocyte sensitization. The anticomplement AHG reagent is generally not used in antibody screening or crossmatch procedures.

\section{Case resolution}

The emergency physician arranges for the patient to be admitted to hospital, and the testing is arranged. The patient's DAT result is strongly positive with anti-IgG AHG reagent only. The eluate prepared from his erythrocytes agglutinates all reagent erythrocytes, a finding that, when combined with the other blood bank findings and the positive biochemical markers of hemolysis, confirms the diagnosis of AIHA.

\section{J. Manuel Zarandona}

Residency Training Program

Department of Pathology

University of Pittsburgh

Pittsburgh, Pa.

Mark H. Yazer

Medical Director

RBC Serology Reference Laboratory

Centralized Transfusion Service

Institute for Transfusion Medicine

Department of Pathology

University of Pittsburgh

Pittsburgh, Pa.

Acknowledgements: We thank Dr. Darrell Triulzi for his thoughtful discussion and critical review of the manuscript.

Competing interests: None declared.

\section{REFERENCES}

I. Brecher ME, editor. American Association of Blood Banks technical manual. I4th ed. Bethesda, MD: American Association of Blood Banks Press; 2002.

2. Freedman J. The significance of complement on the red cell surface. Transfus Med Rev ig87; 58-70.

\section{LEADERSHIP}

$C M A J$ is a founding member of the International Committee of Medical Journal Editors, an organization that is devoted to ensuring the highest integrity in scientific publishing and is a driving force in the mandatory registration of clinical trials. 